\title{
Theoretical and Experimental Analysis of DOP Used as Control Signal in PMD Compensation
}

\author{
Xiangye Zeng ${ }^{1}$, Jianfei Liu ${ }^{1}$, Jian Wang ${ }^{2}$ \\ ${ }^{1}$ School of Information Engineering, Hebei University of Technology, Tianjin 300401, P.R. China \\ ${ }^{2}$ Laboratory of Fiber Optic Communications, School of Electronics Information Engineering \\ Tianjin University, Tianjin 300072, P.R. China
}

\begin{abstract}
Since polarization mode dispersion (PMD) is a major impairment for high bit rate fiber optics systems, it is an important thing for system performance that an proper designed PMD compensate component. In orderto use degree of polarization (DOP) as the feedbacd control signal in PMD dynamic compensation, the relationship beween DOP and differential group delay (DGD) must be ascertain first. Firstly, with a mono-chromatic assumption, the mathematics expression ofDOP is derived in this paper. Then, the relationship beween DOP and DGDm pulse width, and power splitting ratio is analyzde when optic pulse is Gaussian. At last, an experiment is presented, in which 10Gbit/s RZ pseudo random sequence is adopted, and the experimental results prove the validity of theoretical analysis.
\end{abstract}

Keywords: degree of polarzation (DOP), polarization mode dispersion (PMD) , PMD dynamic compensation, differential group delay (DGD)

\section{Introduction}

Polarization mode dispersion (PMD) is a crucial limitation in optical communication systems, so PMD compensation component become the major problem of system design. Several feedback control signal extracting schemes of PMD dynamic compensation have been proposed[1], among which the scheme with degree of polarization (DOP) as control signal become more and more arrestive for its unique character[1, 2]. This scheme is a classic optics compensation scheme. Figure 1 shows its principle diagram. ${ }^{1}$

As shown in Figure 1, DOP analyze module gives the DOP information which follows the DGD variety of fiber line. After processing of the DOP information, the compensation algorithm gives the control signal of polarization Controller (PC) and

${ }^{1}$ Corresponding author.

Email address: zengxy@hebut.edu.cn
DGD line, which makes the DOP of DOP analyze module output signal increase, accordingly, decrease the DGD of output signal.

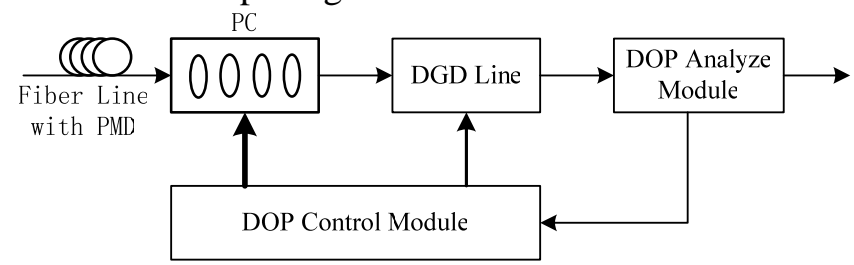

Figure.1 PMD compensation scheme with DOP as the feedback control signal

Since DOP is independent of bit rate of optic signal in fiber line[3, 4], i.e. bit rate transparency, the compensation scheme mentioned before has not the bit rate limitation of fiber optics communication. And DOP analysis do not require high speed optic/electronic detector, this will reduce system cost remarkable. Therefore, optical DOP as feedback control signal has a nice prospect in PMD dynamic compensation. 
The key point of the compensation scheme mentioned before is generating control signal from DOP information. Consequently, it is necessary to study the relation between DOP and DGD of fiber line. In this paper, the mathematical expression of the relation between DOP and DGD is derived, an experiment is presented, in which 10Gbit/s RZ pseudo random sequence is adopted. The experimental results show good coherence with theoretical analysis.

\section{Theory}

It is well known that DOP can be described as

$$
D O P=\frac{I_{p o l}}{I_{p o l}+I_{\text {unp }}},
$$

where $I_{p o l}$ and $I_{\text {unp }}$ are the intensity of polarized and unpolarized light respectively. The value of DOP is between 0 and 1, while 1 represents absolutely polarized light and 0 represents unpolarized light.

DOP can also be described as

$$
D O P=\frac{\sqrt{S_{1}^{2}+S_{2}^{2}+S_{3}^{2}}}{S_{0}},
$$

where (S0; S1; S2; S3) is the Stokes vector. Remember that Stokes vector is a function of angular frequency $\omega$ when optical signal is propagating in fiber with PMD.

\subsection{Relation between DOP and Correlation Function}

Polarization state of quasi monochromatic light is based on the coherent matrix, which can be described by Jones matrix. A 2 orders coherent matrix can be described as

$$
J=\left\langle E_{x y}(t) \times E_{x y}^{*}(t)\right\rangle,
$$

that is

$$
J=\left(\begin{array}{ll}
\left\langle E_{x} E_{x}^{*}\right\rangle & \left\langle E_{x} E_{y}^{*}\right\rangle \\
\left\langle E_{y} E_{x}^{*}\right\rangle & \left\langle E_{y} E_{y}^{*}\right\rangle
\end{array}\right)=\left(\begin{array}{ll}
J_{x x} & J_{x y} \\
J_{y x} & J_{y y}
\end{array}\right)
$$

where $E_{x}$ and $E_{y}$ are the $\mathrm{x}, \mathrm{y}$ directions' electric vectors of Jones matrix respectively, and $J_{x x}=\left\langle E_{x} E_{x}^{*}\right\rangle, J_{x y}=\left\langle E_{x} E_{y}^{*}\right\rangle, J_{y x}=\left\langle E_{y} E_{x}^{*}\right\rangle$, $J_{y y}=\left\langle E_{y} E_{y}^{*}\right\rangle$. Symbol $\langle\cdot\rangle$ represents time average, i.e. $\frac{1}{T} \int_{0}^{T}\langle\cdot\rangle d t$.

$J_{x x}$ and $J_{y y}$ are both real numbers, and they represent the $\mathrm{x}$ and $\mathrm{y}$ polarization components of optical pulse, i.e. $I_{x}=J_{x x}, I_{y}=J_{y y}$, therefore the total light intensity $I$ can be expressed as

$$
I=I_{x}+I_{y}=J_{x x}+J_{y y}=\left\langle E_{x} E_{x}^{*}\right\rangle+\left\langle E_{y} E_{y}^{*}\right\rangle .
$$

$J_{x y}$ and $J_{y x}$ represent the cross-correlativity of $\mathrm{x}$ and $\mathrm{y}$ polarization components, and they are complex conjugates.

Stokes parameters can be expressed by the elements of Jones matrix as follow,

$$
\begin{aligned}
& S_{0}=J_{x x}+J_{y y}, \\
& S_{1}=J_{x x}-J_{y y}, \\
& S_{2}=J_{x y}+J_{y x}, \\
& S_{3}=-j \cdot\left(J_{x y}-J_{y x}\right) .
\end{aligned}
$$

Then, by substitution of equation (6), (7), (8) and (9) into equation (2), we get

$$
D O P=\sqrt{1-\frac{4 I_{x} I_{y}}{\left(I_{x}+I_{y}\right)^{2}}\left(1-\frac{J_{x y} J_{y x}}{I_{x} I_{y}}\right)} .
$$

Let $\mu_{x y}=\frac{J_{x y}}{\sqrt{I_{x} \cdot I_{y}}}$ and $\mu_{y x}=\frac{J_{y x}}{\sqrt{I_{x} \cdot I_{y}}}$, then

$$
\mu^{2}=\mu_{x y} \cdot \mu_{y x}=\frac{J_{x y} J_{y x}}{I_{x} \cdot I_{y}},
$$

$$
\mu=\sqrt{\frac{J_{x y} J_{y x}}{I_{x} \cdot I_{y}}},
$$

where $\mu$ is called normalized cross-correlation function. Then DOP can be expressed as

$$
D O P=\sqrt{1-\frac{4 I_{x} I_{y}}{\left(I_{x}+I_{y}\right)^{2}} \cdot\left(1-\mu^{2}\right)} .
$$

Equation (13) is the DOP of optical signal expressed by light intensity and normalized cross-correlation function. From equation (13), we can see obviously that DOP is not only related to light intensity of $\mathrm{x}, \mathrm{y}$ directions, but also related to normalized cross-correlation function $\mu$.

For $J_{x y}$ and $J_{y x}$ are complex conjugates, i.e. $J_{x y}=J_{y x}^{*} \quad, \quad$ then we get $\mu^{2}=\mu_{x y} \mu_{y x}=\mu_{x y} \mu_{x y}^{*}=\mu_{y x} \mu_{y x}^{*}$.

\subsection{Relation between DOP and DGD}

Because of the effect of fiber PMD, optical pulse will have some time delay $\Delta \tau$ between $\mathrm{x}$ and $\mathrm{y}$ direction after a long distance transmission. For mathematics facility, in derivation, we use $\Delta \tau$ to 
represent DGD. Let the waveform function of optical pulse be $f(t)$ and power splitting ratio of two perpendicular directions be $\gamma$, then $\mathrm{x}$ and $\mathrm{y}$ directions' polarized components of optical pulse can be expressed as

$$
\begin{gathered}
E_{x}=\sqrt{\gamma} f(t) e^{j\left(\omega t+\delta_{x}\right)} \\
E_{y}=\sqrt{1-\gamma} f(t-\Delta \tau) e^{j\left(\omega t+\delta_{y}\right)} \\
\text { By substitution of equation (14) and (15) into } \\
J_{x y}=\left\langle E_{x} E_{y}^{*}\right\rangle \text {, we can get } \\
J_{x y}=\left\langle E_{x} E_{y}^{*}\right\rangle=\frac{1}{T} \int_{0}^{T}\left[\sqrt{\gamma} f(t) e^{j\left(\omega t+\delta_{x}\right)}\right] \cdot\left[\sqrt{1-\gamma} f(t-\Delta \tau) e^{-j\left(\omega t+\delta_{y}\right)}\right] d t
\end{gathered}
$$

Then substitute for the $J_{x y}$ in equation (11), we get the expression of normalized cross-correlation function as follow,

$$
\mu^{2}=\frac{\left[\int_{0}^{T} f(t) f(t-\Delta \tau) d t\right]^{2}}{\int_{0}^{T} f^{2}(t) d t \cdot \int_{0}^{T} f^{2}(t-\Delta \tau) d t}
$$

Where the denominator is the time integral of the square of waveform function $f(t)$ in $[0, \mathrm{~T}]$ time segment, that is the energy of optical pulse, so it should be a constant. When $\mathrm{T}$ is long enough, the energy of delayed $f(t)$ will be a constant either.

By substitution of equation (16) into equation (13), and considering splitting ration $\gamma, I_{x}=\gamma I$ and $I_{y}=(1-\gamma) I$, we can get

$$
D O P=\sqrt{1-4 \gamma(1-\gamma) \cdot\left(1-\frac{\left(\int_{0}^{T} f(t) f(t-\Delta \tau) d t\right)^{2}}{\int_{0}^{T} f^{2}(t) d t \cdot \int_{0}^{T} f^{2}(t-\Delta \tau) d t}\right.}
$$

Equation (17) is the mathematics expression of DOP, i.e. the relation between DOP and DGD $(\Delta \tau)$. We can see from equation (17) that DOP is not only related to DGD ( $\Delta \tau)$, but also related to waveform function $f(t)$ and power splitting ratio $\gamma$.

\subsection{Relation between DOP and DGD for Gaussian Pulse}

Now assume that the waveform function of optical pulse transmitted in optic fiber is Gaussian, that is

$$
f(t)=e^{-\frac{t^{2}}{2 \sigma_{0}^{2}}}
$$

Where $\sigma_{0}$ is the half width at $1 / e$ maximum amplitude of optical pulse. But full width at half maximum (FWHM) is a practical representation of pulse width. For Gaussian pulse, the relation between them is

$$
T_{\text {FWHM }}=2 \sqrt{\ln 2} \sigma_{0}=1.665 \sigma_{0}
$$

Keep in mind this relation, we will use it when analyzing the experimental results.

By substitution of equation (18) into equation (17), we can get

$$
D O P=\sqrt{1-4 \gamma(1-\gamma) \cdot\left(1-\frac{e^{\frac{\Delta \tau^{2}}{2 \sigma_{0}}}}{2} \cdot \frac{\left[\operatorname{erf}\left(\frac{T_{0}+\Delta \tau}{2 \sigma_{0}}\right)+\operatorname{erf}\left(\frac{T_{0}-\Delta \tau}{2 \sigma_{0}}\right)\right]^{2}}{\operatorname{erf}\left(\frac{T_{0}}{\sigma_{0}}\right) \cdot\left[\operatorname{erf}\left(\frac{T_{0}+2 \Delta \tau}{2 \sigma_{0}}\right)+\operatorname{erf}\left(\frac{T_{0}-2 \Delta \tau}{2 \sigma_{0}}\right)\right]}\right.}
$$

Equation (20) is the relation between DOP and DGD ( $\Delta \tau)$, splitting ratio $\gamma$, and pulse width $\sigma_{0}$ for Gaussian pulse.

From equation (20), we can get the theoretical curve as figure 2 and figure 3 .

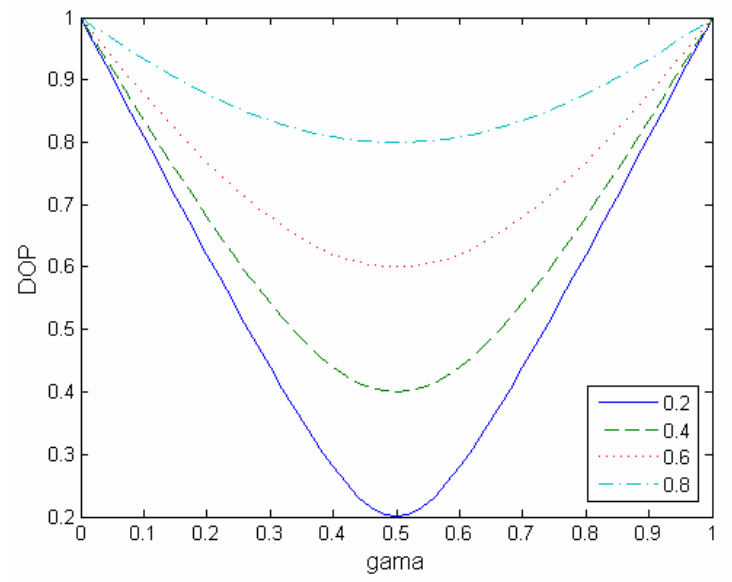

Figure.2 Relation curve between DOP and splitting ratio $\gamma$ under different normalized cross-correlation function $\mu$

Figure 2 is the DOP curve varying with splitting ratio when normalized cross-correlation function $\mu$ takes value $0.2,0.4,0.6$ and 0.8 respectively. Figure 2 shows that DOP takes the minimum value when $\gamma$ equals 0.5 and takes 1 when $\gamma$ equals 0 or 1 . This relation is consistent with qualitative analysis.

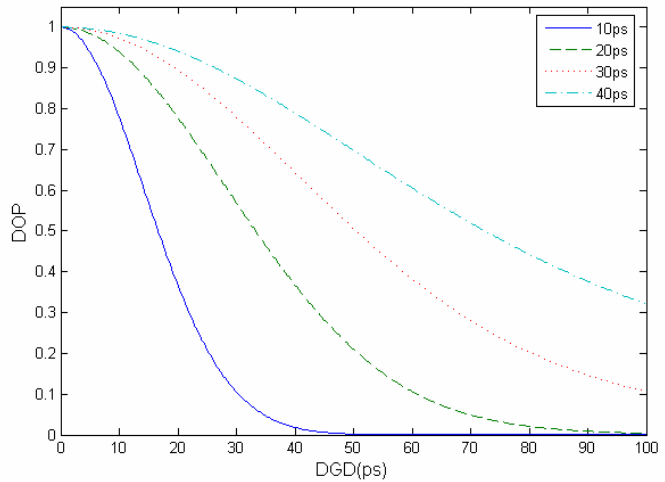

Figure.3 Relation curve between DOP and DGD $(\Delta \tau)$ under different pulse width $\sigma_{0}, \gamma=0.5$ 
Figure 3 is the DOP curve varying with DGD $(\Delta \tau)$ when splitting ratio $\gamma$ equals 0.5 and pulse width $\sigma_{0}$ takes value $10 \mathrm{ps}, 20 \mathrm{ps}$, 30ps and 40ps respectively. Figure 3 shows that DOP has a monotone decreasing relation with DGD in the definite time range, so DOP can be used as the feedback control signal of PMD dynamic compensation. We can also see in figure3 that the curve become more steep while the pulse width $\sigma_{0}$ decreasing. This means that compensation method using DOP as feedback control signal will have a decreasing dynamic compensation range while using in high bit rate system.

\section{Experimental Results}

We study the relation between DOP and DGD by experimental method as follow. Experimental device is shown in figure 4.

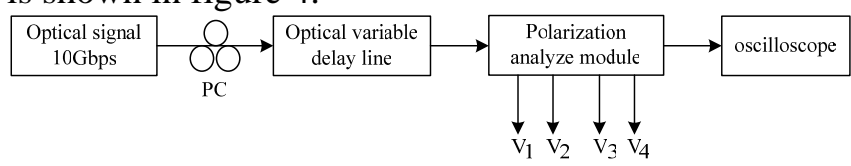

Figure.4 Experimental device to measure DOP

Figure 4 shows the experimental device for DOP measurement. Where optical signal is 10Gbit/s RZ pseudo random sequence. Light polarization controller (PC) changes the polarization direction of optical signal which enters the polarization analyze module, thus produces the splitting ratio $\gamma$ we want. Optical variable delay line provides the time delay $(\Delta \tau)$ we need. With the variation of delay time, the variation of polarization state is measured., and DOP can be calculated. Eye diagram can be observed in oscilloscope, and corresponding relation between DOP and eye diagram can be obtained.

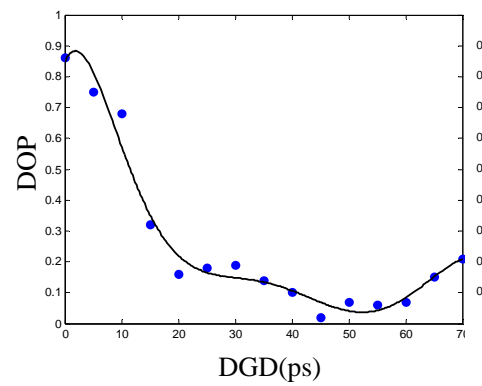

(a)

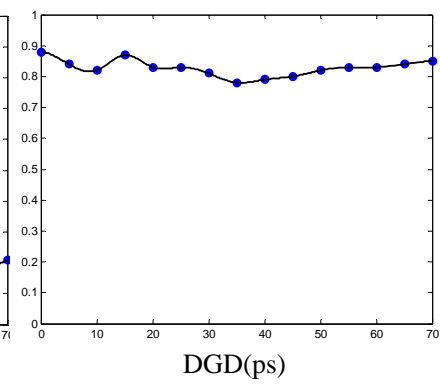

(b)
Figure.5 Measurement results of 10ps optical pulse

Figure 5 shows the measurement curve of DOP by DGD when pulse width (FWHM) equals 10ps.
The splitting ratio $\gamma$ of figure $5(\mathrm{a})$ is about 0.5 , and that of figure $5(\mathrm{~b})$ is about 0 or 1 .From these figures, we can see that DOP of figure 5(a) is obviously small than DOP of figure $5(\mathrm{~b})$, which is consistent with the theoretical analysis in figure 2 .

According to equation (19), pulse width $\sigma_{0}$ in experiment is about 6ps. Compared figure 5(a) with figure 3 , we can see that measurement curve is basically same as the 10ps curve in figure 3 when DGD is less than 60ps. When DGD is greater than $60 \mathrm{ps}$, DOP is increasing in measurement curve and can not agree with theoretical curve. After analysis, we think that this is caused by the overlapping of the slow component of former pulse and the fast component of later pulse when DGD is great enough.

The measurement curve of DOP by DGD is shown in figure 6, when pulse width (FWHM) increased to 43ps and splitting ratio kept constant.

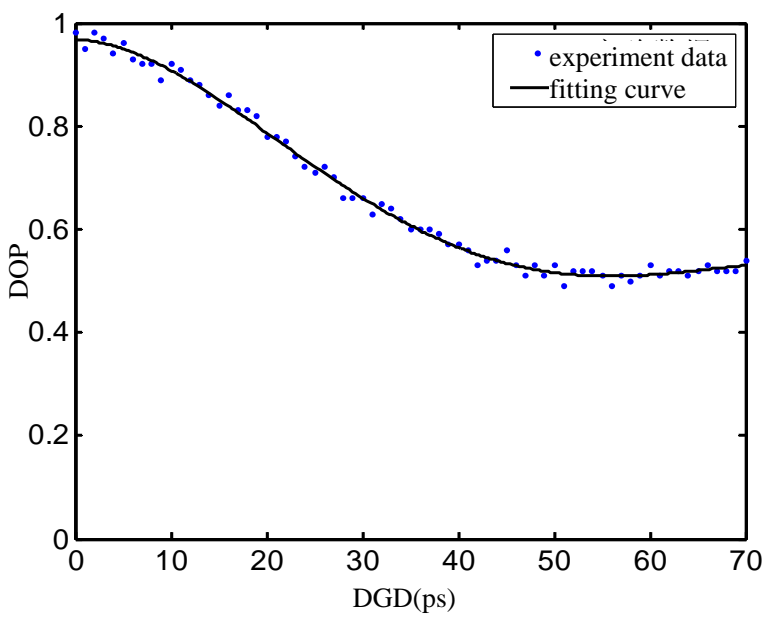

Figure.6 Measurement results of 43ps optical pulse

Figure 6 shows the fitting curve of measured data in solid line. Compared with figure 5 , we can see that DOP presents monotone decreasing relation with DGD in a larger time range when pulse width is broadened.

According to equation (19), pulse width $\sigma_{0}$ in experiment is 25.8ps. Compared with figure 3, we can see that measurement curve is basically same as the 30ps curve in figure 3.

In order to demonstrate the consistency, we compare the theoretical analysis and experimental results of optical pulse with 10ps and 43ps pulse width (FWHM). Splitting ratio equals 0.5 while theoretical analyzing.

Figure 7 shows theoretical curve and experiment data comparison of 10ps optical pulse. From figure 7, 
we can see that measurement data is consistent with theoretical curve very well when DGD is less than 20ps, which prove the validity of theoretical analysis in this time range. But experiment data appear second peak, which is not consistent with theoretical curve. After analysis, we attribute it to the chirp of original pulse.

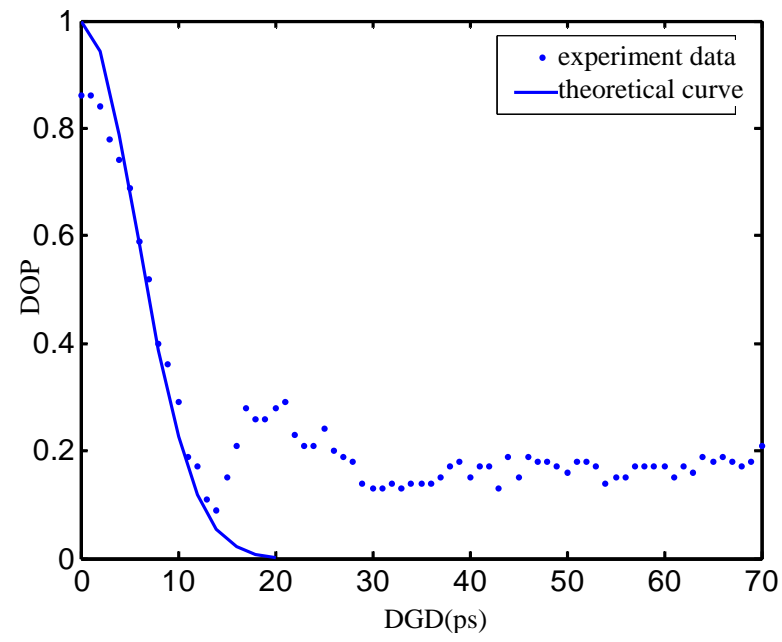

Figure.7 Comparison of theoretical curve and experiment data of 10ps optical pulse

Short pulse, like 10ps, is generated by pulse compression, and pulse compression will introduce chirp in pulse, which generates secondary pulse. At the time position secondary pulse appears, corresponding normalized cross-correlation function will increase, which will result in the increase of DOP.

Figure 7 shows theoretical curve and experiment data comparison of 10ps optical pulse. From figure 7 , we can see that measurement data is consistent with theoretical curve very well when DGD is less than 20ps, which prove the validity of theoretical analysis in this time range. But experiment data appear second peak, which is not consistent with theoretical curve. After analysis, we attribute it to the chirp of original pulse. Short pulse, like 10ps, is generated by pulse compression, and pulse compression will introduce chirp in pulse, which generates secondary pulse. At the time position secondary pulse appears, corresponding normalized cross-correlation function will increase, which will result in the increase of DOP.

Figure 8 shows theoretical curve and experiment data comparison of 43ps optical pulse. From figure 8, we can see that measurement data is consistent with theoretical curve very well, which prove the validity of theoretical analysis. It is noteworthy that experiment data in figure 8 have no secondary peak like that in figure 7 . The reason for this is that the generation of pulse with width 43ps has no need for pulse compression, then original pulse does not have chirp value, therefore DOP does not appear secondary increase.

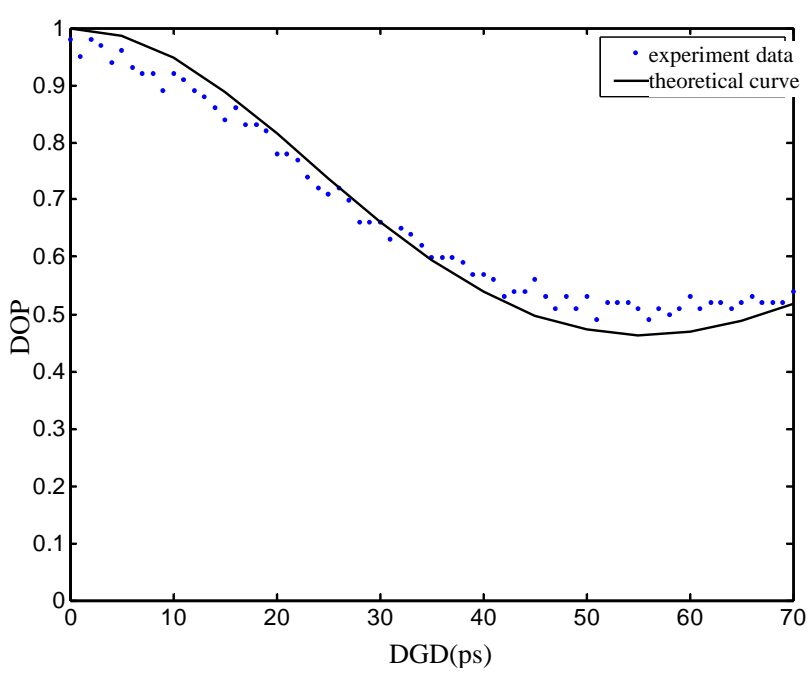

Figure.8 Comparison of theoretical curve and experiment data of 43ps optical pulse

Theoretical analysis and experiment results show that DOP has a definitive relation with DGD, i.e. DOP shows monotone decrease while DGD increases in the definite time range. DOP is related to splitting ratio $\gamma$ along two principle states of polarization (PSP). When other conditions fixed, the more splitting ratio $\gamma$ is close to 0.5 , the greater the variation of DOP becomes. DOP is related to pulse width, too. The greater the pulse width is, the smaller the variation of DOP becomes. All the conclusions above obtains from theoretical and experimental analysis, and experiment results are basically consistent with theoretical analysis.

\section{Conclusions}

Based on mono-chromatic assumption, the mathematics relation between DOP and DGD of optical pulse transmitted in optic fiber is derived in this paper. The detail DOP expression of Gaussian pulse is obtained. Experiment data are given based on an experiment using 10Gbit/s RZ pseudo random sequence with different pulse width, and comparison between theoretical curve and experiment data is done to verify the validity of theoretical analysis.

\section{Acknowledgments}

This work was supported by HENSF of China under Grant No. F2008000116 and HeBei Province educator fund of China under Grant No. 2005354. 


\section{References}

[1] Denis Penninchx and Stephanie Lanne, "Reducing PMD impairments,” In: OFC 2001, pp.TuP-1.

[2] J. Zweck and S. E. Minkoff, "Modeling Compensation for Optical Fiber Communication Systems," SIAM Journal on Optimization, Vol. 17, pp. 738-775, September 2006.

[3] M. F. FERREIRA, A. N. PINTO, P. S. ANDRÉ and N. J. MUGA, "Polarization Mode Dispersion in High-Speed Optical Communication Systems," Fiber and Integrated Optics, Vol. 24, pp. 261-285, 2005.

[4] Y.W. Song, S.M.R. Motaghian, Z. Pan and A.E. Willner, "Efficient DOP monitoring of WDM channels for simultaneous PMD compensation," Optics Communications, Vol. 255, pp. 225-229, 2005.

[5] Pradeep Kumar Kondamuri, "Predicting first-order PMD outage rates on long-haul optical fiber links using measured and modeled temporal and spectral DGD data,” PhD dissertation, University of Kansas, 2002.

[6] Jian Wang, "Researches on Adaptive Technology of Compensation for Polarization Mode Dispersion," PhD dissertation, Tianjin University, 2003.

[7] Hankui Liu, Xianmin Zhang and Kangsheng Chen, "Degree of polarization technique used in PMD compensation of optical microwave transmission systems,” Optics Communications, Vol. 236, pp. 109-114, 2004. 